\title{
Spatio-temporal climate change contributes to latitudinal
}

6

7

8

\section{diversity gradients}

Erin E. Saupe ${ }^{* 1}$, Corinne E. Myers ${ }^{2}$, A. Townsend Peterson ${ }^{3}$, Jorge Soberón ${ }^{3}$, Joy

Singarayer $^{4}$, Paul Valdes ${ }^{5}$, Huijie Qiao*6

${ }^{1}$ Department of Earth Sciences, University of Oxford, South Parks Road, Oxford OX1 3AN, UK

${ }^{2}$ University of New Mexico, Northrop Hall, Albuquerque, New Mexico 87131, USA

${ }^{3}$ Biodiversity Institute, University of Kansas, Lawrence, KS 66045, USA

${ }^{4}$ Department of Meteorology and Centre for Past Climate Change, University of Reading, Reading, UK

${ }^{5}$ School of Geographical Sciences, University of Bristol, Bristol, UK

${ }^{6}$ Institute of Zoology, Chinese Academy of Sciences, 1 Beichen West Road, Chaoyang District, Beijing 100101, China

*Corresponding authors: erin.saupe@earth.ox.ac.uk \& qiaohj@ioz.ac.cn

\section{Abstract}

The latitudinal diversity gradient (LDG), in which the number of species increases from the poles to the Equator, ranks among the broadest and most notable biodiversity patterns on Earth. The pattern of species-rich Tropics relative to species-poor temperate areas has been recognized for well over a century, but generative mechanisms are still debated vigorously.

We use simulations to test whether spatio-temporal climatic changes could generate largescale patterns of biodiversity as a function of only three biological processes - speciation, extinction, and dispersal—omitting adaptive niche evolution, diversity-dependence, and 
coexistence limits. In our simulations, speciation resulted from range disjunctions, whereas extinction occurred when no suitable sites were accessible to species. Simulations generated clear LDGs that closely match empirical LDGs for three major vertebrate groups. Higher tropical diversity primarily resulted from higher low-latitude speciation, driven by spatiotemporal variation in precipitation rather than in temperature. This suggests that spatiotemporal changes in low-latitude precipitation prompted geographic range disjunctions over Earth history, leading to high rates of allopatric speciation that contributed to LDGs. Overall, we show that major global biodiversity patterns can derive from interactions of species' niches (fixed a priori in our simulations) with dynamic climate across complex, existing landscapes, without invoking biotic interactions or niche-related adaptations. gradient (LDG) ranks amongst the most striking features of life on Earth ${ }^{1}$. The LDG has been observed in both terrestrial ${ }^{2,3}$ and marine $e^{4,5}$ realms, in plants ${ }^{6,7}$ and animals ${ }^{8,9,10}$, and to varying degrees throughout geological time ${ }^{11,12,13}$. However, despite over a century of study and analysis, consensus on causal mechanisms has yet to be reached ${ }^{1,3,14,15,16}$.

In the simplest sense, LDGs can be explained only by particular combinations of differential speciation rates (Tropics as a cradle; ${ }^{17,18,19}$ ), extinction rates (Tropics as a museum; ${ }^{18,20,21}$ ), and/or dispersal rates into the Tropics ${ }^{22,23}$. The causal mechanisms behind such differential rates ${ }^{8,10,15,24}$, however, remain uncertain. Although over 40 ecological and evolutionary explanations have been proposed ${ }^{1,14,15,25}$, most researchers agree that a nearubiquitous pattern should have a consistent mechanistic explanation applicable across different life histories and habitats ${ }^{26}$. 
Here, we explore whether spatio-temporal climate dynamics can produce elevated diversity at low latitudes, offering a simple explanation for LDGs. We propose that fluctuating climate conditions yield higher rates of range fragmentation at low latitudes compared to high latitudes $27,28,29,30,31$. Range fragmentation isolates populations, which results in allopatric speciation if populations remain separated for sufficiently-long periods of time. Isolation and speciation may be facilitated by species' inability to adapt quickly to changing climatic conditions ${ }^{23,32,33,34,35,36}$ (Fig. 1). Our specific hypothesis, then, is that spatio-temporal dynamics of climate change generate higher rates of allopatric speciation at low latitudes, contributing to LDGs.

We follow recent work ${ }^{22,28,29,31,37,38,39}$ to extend process-based simulations to terrestrial areas globally ${ }^{40,41}$. Rangel et al. ${ }^{29}$ showed that such simulations can replicate many real-world biodiversity patterns, and tested the role of orographic features such as the Andes in structuring biodiversity spatially. We diverge from that work ${ }^{29}$ by specifying in our simulations only two species traits— niche breadth and dispersal ability—and incorporating only influences of the abiotic environment, without invoking additional processes such as biotic interactions or niche evolution ${ }^{9,23,32,42}$. These simplifying assumptions were made to explore the sufficiency of abiotic processes in explaining observed biodiversity patterns ${ }^{26}$. Using process-based, spatially- and temporally-explicit simulations ${ }^{40,41,43}$ from the Eemian (last interglacial) to present, we recorded the spatial pattern of diversity generated at the end of each simulation (Table 1). We additionally noted the location and frequency of the speciation, extinction, and dispersal events that generated this biodiversity, to explore their relative contributions to LDGs and the potential climatic drivers behind these macroevolutionary dynamics.

\section{Results}


Mean latitudinal diversity was quantified by selecting one simulation from each $1^{\circ}$ latitudinal band in a series of 100 bootstrap replicates. As a consequence, global diversity was initially equivalent across latitudes (i.e., one 'species' per latitudinal band) at the start of simulations. However, when diversity was quantified from selected seeds after simulations completed, species diversity was maximum at the Equator and lower at higher latitudes (Fig. 2). This pattern was most pronounced among species with narrow niches and poor dispersal abilities, and under climate scenarios in which ice sheets were included as biogeographic constraints (Fig. 2; Figs. S1-4). Species with better dispersal ability and/or broader niches showed flattened latitudinal diversity curves, and we even noted a middle-latitude diversity bump in scenarios in which ice sheets were not considered (Figs. S1, S5 \& S6). Scaling species numbers to area available in latitudinal bands steepened the LDGs (Figs. S7-12) and removed the higher-latitude peaks for species with broad ecological niches (Figs. S9 \& S10). Simulated New World diversity provided much of the elevated low-latitude diversity for the global LDG. Higher tropical diversity was observed under all scenarios in the New World (Figs. S13-15), though the most dramatic LDG resulted from species with poor dispersal abilities and narrow niche breadths (Fig. S15). Europe and Africa showed more dampened LDGs, with higher tropical diversity only among species with poor dispersal abilities and/or narrow niche breadths. Asia and Australia were characterized by reverse gradients in all scenarios, with higher diversity at higher latitudes. Scaling numbers of species on a per-unit-area basis in latitudinal bands resulted in yet-steeper New World diversity gradients (Figs. S16-18), and higher-latitude diversity peaks in broad-niched species were dampened or eliminated.

To evaluate the role of climate change versus time-independent spatial climate variation alone, we ran a series of simulations in which climate was held constant. These 
simulations did not produce low-latitude peaks in diversity (Figs. S19-21). Results therefore underline the key role of spatial and temporal variation in climate in generating LDGs in our simulations.

109

Rates of speciation and extinction

Speciation rates showed latitudinal patterns that were dependent on interactions between niche breadth and dispersal ability (Fig. 3, Figs. S22-24). At low latitudes, speciation rates were higher for species with narrow niche breadths, regardless of dispersal

114 ability (Fig. 3, Fig. S23); species with broad niches, regardless of dispersal ability, showed

115 the opposite pattern (Fig. S24). This niche-breadth dependency was consistent across climate scenarios. As expected, inclusion of ice sheets dampened high-latitude speciation rates.

117 Scaling to area available in latitudinal bands resulted in significantly higher tropical speciation rates in all niche and dispersal combinations (Figs. S25-27).

Extinction rates were either elevated at high latitudes or similar across latitudes, in all

120 combinations of niche breadth, dispersal ability, and climate scenario (Fig. 3, Figs. S28-30).

121 The only scenario in which extinction rates were higher at lower latitudes $\left(0-8^{\circ} \mathrm{S}\right.$ latitude) was for species with narrow niche breadth and poor dispersal ability (Fig. S30). Scaling to area available in latitudinal bands did not change overall patterns of extinction rates (Figs. S31-33). Speciation outpaced extinction in the simulations, resulting in increased diversity over time (Fig. S34).

Geographic patterns

Geographic patterns of diversity, speciation, and extinction were examined globally on a cell-by-cell basis. We found high levels of diversity equatorially in Brazil and central

130 Africa in all scenarios (Fig. 4, Fig. S35). Southeast Asia, however, was more depauperate. 
131 Simulations returned hotspots of diversity at high latitudes in northern Europe and Asia for

132 broad-niched species (Fig. S35). Diversity hotspots were caused primarily by elevated

133 speciation: high speciation rates coincided with areas of high diversity, whereas extinction

134 rates were low and diffuse throughout terrestrial areas globally (Fig. S35).

Dispersal dynamics

We documented the frequency of shifts from temperate to tropical biomes and vice versa to test the role of dispersal in generating diversity gradients ${ }^{19,44}$. More lineages dispersed from the Tropics to temperate regions than vice versa in our simulations, even

140 when holding the number of species considered constant in each biome ${ }^{45}$. Results were 141 consistent regardless of whether we counted numbers of initial lineages (Fig. 5; Figs. S36-

142 38), total numbers of species dispersing from each initial lineage (Figs. S39-41), or the number of dispersal events from a standardized number of species in each biome (Figs. S4244). The dominance of tropical-to-temperate dispersal over temperate-to-tropical dispersal was statistically significant $(P<0.001$; one-tailed Mann Whitney $U$-tests) in all scenarios.

\section{Climate dynamics}

We assessed temperature and precipitation as potential causal agents in vicariant events separating populations for a sufficiently-long period to induce speciation. Precipitation

150 was responsible for most low-latitude speciation events, regardless of niche or dispersal combination (Fig. 6; Figs. S45-S47). Temperature, however, became an equally-important driver of speciation around $30-35^{\circ} \mathrm{N}$, and a more important driver by around $50-55^{\circ} \mathrm{N}$.

153 Extinction was caused by changes in both temperature and precipitation, although the relative 154 contribution of temperature was higher at high latitudes (Fig. 6; Figs. S48-S50). 

implicate precipitation as the primary driver of elevated low-latitude speciation. The magnitude of precipitation change was greater at low latitudes over the last $120 \mathrm{Ka}$, whereas the magnitude of temperature change was greater at high latitudes over the same time period.

159 This relationship can be quantified by correlating latitude with the mean standard deviation of 160 climate change amongst cells within those latitudes over the last $120 \mathrm{Ka}$; the relationship was negative for precipitation $(r=-0.80)$, but positive for maximum and minimum temperature $(r$ $=0.65$ and 0.83 , respectively). This pattern indicates that variation in precipitation decreases with latitude, but variation in both maximum and minimum temperature increases with

164 latitude.

165 Temporal changes in precipitation were spatially heterogeneous at low latitudes. That is, some equatorial regions experienced significant change in precipitation over the last 120

167 Ka, whereas adjacent regions experienced relatively little change. This heterogeneity is illustrated by significant peaks and troughs in the standard deviation of precipitation values at

169 low latitudes (Fig. S51a). Conversely, temperature did not fluctuate significantly over the last

$170120 \mathrm{Ka}$ in equatorial regions, but did change at high latitudes. Unlike precipitation, these high-latitude temporal fluctuations were not spatially heterogeneous but occurred over continuous and broad regions (Fig. S51b,c).

\section{Comparisons with empirical patterns}

We calculated cell-by-cell Pearson correlation coefficients $(r)$ between simulated diversity and empirical diversity for three clades (birds, mammals, amphibians) using standardized, global diversity maps (Table S1). In most cases, our simulated diversity patterns correlated positively with diversity patterns for birds, mammals, and amphibians; negative correlations resulted in only a few cases in simulations considering broad-niched 
species (Table S1). Strongest correlations were recovered for simulations using species with narrow niches and poor dispersal abilities ( $r^{2}>0.16$ for mammals). Correspondence between simulated diversity and bird and mammal diversity was higher than expected based on null distributions generated by reshuffling simulated diversity values within each latitudinal band in a series of 100 replicates (Fig. S52 and Table S2). Correlations were weaker than expected for amphibians when considering species with broad niches and poor dispersal abilities (Table S2).

Simulated diversity was subtracted from empirical diversity on a cell-by-cell basis to examine regions where simulations over- or under-predicted diversity. Diversity patterns were replicated closely across broad swaths of global terrestrial regions. However, our

190 simulations generally tended to under-predict diversity in South America and over-predict diversity in central Africa (Fig. S53). Europe and Asia tended to be characterized by too-high simulated diversity for species with broad niches. using Fréchet distances, which measures similarity between two curves while considering the location and ordering of the points along the curves. Simulated latitudinal diversity curves were remarkably similar to empirical diversity curves, although high-latitude diversity in the Northern Hemisphere was over-predicted for species with broad niches (Figs. 2; Figs. S5-6). Simulated diversity curves were most similar to empirical diversity curves when considering species with narrow niches and poor dispersal abilities, and most dissimilar when considering species with broad niches regardless of dispersal ability (Table S3). Overall, simulated diversity curves were most similar to the mammal diversity curve (Fig. 4 and Table S3). Indeed, we found shorter Fréchet distances between mammals and simulated diversity using species with narrow niches, than when comparing mammals, birds, and amphibians to each

204 other. Considering all niche and dispersal combinations together, Fréchet distances from our 
simulations to mammals or birds were usually no greater than the distance between birds and amphibians (Table S3), suggesting that the simulations are closely replicating large-scale attributes of empirical diversity patterns.

We also assessed similarity between geographic range-size frequency distributions in our simulations and those of birds, mammals, and amphibians. As in empirical range-size

210 frequency distributions $s^{46,47,48}$, simulated range-size frequency distributions were right-

211 skewed (Fig. S54). Although range-size frequency distributions were within the same general

212 magnitude as empirical groups, simulated species tended to have larger geographic ranges.

213 Consequently, the distribution for simulated species for all niche $\times$ dispersal $\times$ climate

214 scenario combinations differed significantly from empirical distributions based on

215 Kolmogorov-Smirnov two-sample tests $(p$-value < 0.001). However, range-size frequency

216 distributions for empirical distributions also differed significantly $(p<0.001)$ from one

217 another.

219 Discussion

We simulated a world in which the three factors affecting biological diversityspeciation, extinction, and dispersal—responded only to realistic climate dynamics over the terrestrial global landscape. Starting from no diversity gradient, the great majority of our simulations yielded higher diversity at low latitudes, particularly for species with narrow

224 niches. This result is consistent with previous, independent sets of simulations that employed

225 similar frameworks for South America ${ }^{28,29}$. Speciation rates, rather than extinction rates, were the primary control on these patterns, being markedly elevated in the Tropics, which is congruent with previous findings that suggest the Tropics as a cradle of biological diversity ${ }^{10,}$ 15, 19, 24, 49 . Dispersal into the Tropics did not contribute significantly to the strength of the LDG, since species dispersed from lower to higher latitudes at higher rates under all dispersal 
and niche combinations; in this sense, our simulations supported the 'out of the Tropics' model $^{19,44,50}$. Given that species diversity scales with area ${ }^{51,52}$, and that land area is reduced at lower latitudes, rescaling diversity curves to available area further amplified low-latitude peaks in diversity for all niche and dispersal scenarios, and eliminated peaks in diversity at higher latitudes for species with broad niches. Results were not dependent on the presence or absence of continental ice sheets.

Simulated diversity was consistent with the well-documented, empirical patterns of steeper diversity gradients and higher overall diversity in the New World ${ }^{2}$ and in species with poor dispersal abilities ${ }^{53}$. This outcome is remarkable, since no target diversity pattern was sought, and we started each simulation with a world with only one species per latitudinal band. Moreover, simulations replicated the shape of empirical LDG curves with high fidelity, including a northern latitude diversity drop at $\sim 20^{\circ}$ that has been documented in empirical studies (Fig. 4) $)^{54,55}$, and higher numbers of specialist species at low latitudes ${ }^{16,56,57}$ (Fig. S55). Simulated diversity patterns diverged most from those of amphibians, which is unsurprising, given that amphibians typically have narrow niches and low dispersal potential, probably lower than that in our simulations ${ }^{58,59}$. Amphibians possess a mean latitudinal range of $\sim 4.2^{\circ}$, compared to $12.4^{\circ}, 21.9^{\circ}$, and $22.2^{\circ}$ for mammals, our aggregate simulations, and birds, respectively.

Our simulated biodiversity patterns and real-world diversity hotspots were similar, at

249 least at coarse scales, except for Southeast Asia, Australia, and New Guinea (Fig. 4).

250 Observed differences between simulated and empirical patterns may reflect model misspecification, or more ancient (pre-Eemian) processes not integrated into our simulations, such as tectonic changes and environmental perturbations. Geographic range-size frequency distributions also replicated the right-skewed nature of empirical distributions, except that a

254 greater proportion of simulated species had larger ranges. This skew is not surprising, since 
virtual species were accorded relatively broad niches (i.e., $21^{\circ}$ and $31^{\circ} \mathrm{C}$ temperature breadth for narrow and broad niche species, respectively) that were not limited by biotic interactions.

257 Larger ranges, however, should not affect interpretations of simulated results or LDG mechanisms in a systematic fashion. Indeed, reduction in species' range sizes, either from narrowing tolerance limits or from biotic interactions, may further facilitate climate-driven tropical range fragmentation and more effective isolation of those range fragments (increasing speciation potential).

Analysis of the spatio-temporal dynamics of precipitation and temperature and their relationships to speciation and extinction revealed that tropical diversity was primarily a byproduct of precipitation (Fig. 6). Patterns of precipitation are spatially and temporally heterogeneous at low latitudes (Fig. S51) ${ }^{60,61,62,63}$, and this heterogeneity served to fragment species' ranges, resulting in isolation and eventual lineage splitting. Extinction, however, was caused by variation in temperature and precipitation across latitudes, although the relative contribution of temperature to extinction was higher at high latitudes (Fig. 6). High-latitude extinction driven by Cenozoic cooling may further sharpen LDGs ${ }^{10,27,64,65,66}$. This argument is essentially equivalent to the 'Tropics as older' hypothesis, which proposes that climatic stability at low latitudes resulted in dampened extinction rates at low latitudes ${ }^{50,67,68}$. Diversity peaks were obtained at high latitudes for species with broad niches, with temperature (Fig. 6). Wider and more continuous temperature barriers at high latitudes (Fig.

275 S51) were able to isolate effectively generalist species, but were less effective at fragmenting ranges of species with narrow niches. That is, spatio-temporal temperature changes at high

277 latitudes were not sufficiently patchy to generate range disjunctions for narrow-niched species, though note that the relative contribution of temperature to speciation was high at high latitudes for both narrow- and broad-niched species. 

heterogeneity on diversification concluded that temperature, rather than precipitation, drove speciation and extinction. Rangel and colleagues ${ }^{29}$ examined correlations between mean annual climate values and mean speciation rates over the South American continent in a timeseries analysis, rather than examining directly the contribution of each climate variable to speciation and extinction in a mechanistic, per-event and spatially-explicit context as we did here (see 'climate dynamics' Methods section). Employing the correlational methodology of Rangel et $\mathrm{al}^{29}$, we obtain a significant role for precipitation and also for temperature (Table S4). These divergent patterns may stem from the temporal scale of study (Rangel and colleagues $^{29}$ covered eight glacial-interglacial cycles, whereas our study covered only one), or the inclusion of different processes.

We do not suggest that Eemian (i.e., last glacial cycle) speciation alone is responsible for the modern LDG. The Earth, of course, was already populated by a rich biota at $120 \mathrm{Ka}$. Rather, we hypothesize that interactions between spatio-temporal dynamics of climate, realworld geography, and species operated similarly on geologic timescales ${ }^{31,69,70}$, which could have contributed to the maintenance of LDGs observed today. This effect would explain the correspondence of our simulated biodiversity patterns to empirical biodiversity patterns. Importantly, our hypothesis can be further interrogated as new and more dense climatic data become available for earlier time periods. Current understanding of paleoclimate dynamics is that they have fluctuated continuously over various timescales ${ }^{71,72,73}$. In our simulations,

300 spatio-temporal heterogeneity in precipitation at low latitudes generated higher rates of 301 allopatric speciation. Although this pattern was derived from Late Pleistocene climate dynamics, low-latitude variability in precipitation has likely occurred throughout much of

303 Earth history, and has been suggested as a driver of hominin evolution in southeastern

304 Africa $^{74}$. Atmospheric moisture is a strongly non-linear function of temperature, meaning that 
small variations in temperature are amplified by the moisture cycle. Consequently, high variability in precipitation is expected in warm climates, such as the low-latitude Tropics ${ }^{63}$. A

307 logical corollary, of course, is that during periods of extreme warmth globally, such as the

308 Late Cretaceous and Early Eocene, precipitation may have been variable everywhere, not

309 only at low latitudes. Based on our proposed mechanism, then, speciation rates would have

310 been high across latitudes, providing a process-based explanation for the dampened diversity

311 gradients often reported for these time periods ${ }^{12,13,75,76,77,78,79}$.

312 Diverse factors have been implicated in producing biodiversity patterns, and

313 particularly the $\mathrm{LDG}^{1,14,15,25}$, and these factors could further enhance and refine spatial

314 patterns of biodiversity. For example, we did not examine effects of niche evolution ${ }^{29}$, area-

315 productivity dynamics ${ }^{80}$, increased biotic interactions and competition ${ }^{29,64,81,82}$, diversity-

316 dependent speciation and extinction processes ${ }^{8}$, or higher energy to accelerate mutation

317 rates $^{83}$ and/or promote species coexistence ${ }^{84}$ on biodiversity patterns. Moreover, whilst mid-

318 domain effects ${ }^{85}$ may contribute to LDGs, they did not contribute significantly to our

319 simulated patterns, because we did not recover diversity gradients in simulations that allowed

320 virtual species to search and occupy suitable areas, but which invoked no climate change ${ }^{86}$

321 (Figs. S19-S21).

The above discussions lead to reflections on the reality and utility of our simulations.

323 Our model is simple, but this simplicity was purposeful and explicitly designed. For example,

324 we invoked no changes in species' niche traits through time or at splitting events. That is, we 325 assumed niche stability during the lifetime of each lineage simulated. Niche stability and

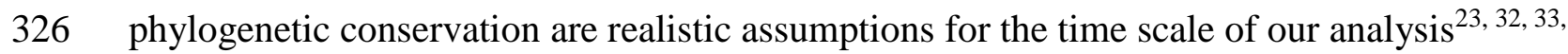
34, 35, 36, and have even been proposed as a mechanism for the modern $\mathrm{LDG}^{9,23,32,42}$.

328 However, evolution in species' niche tolerances does occur over longer time scales and may 329 additionally enhance the $\mathrm{LDG}^{29}$. Furthermore, we invoked no biotic interactions in our 
330 simulations, and we considered only a set of trait values for dispersal and niche breadth:

331 simulations cast in the context of other dispersal and niche traits may show different patterns.

332 Although biotic interactions could contribute to LDGs ${ }^{8,64,81,82}$, we do not consider them here

333 for two reasons: (i) to examine the extent to which abiotic factors alone offer a mechanism to

334 explain observed patterns of diversity; and (ii) because modelled competition was found to

335 have little effect on resulting geographic patterns of species' richness ${ }^{29}$.

336 We assessed terrestrial realms only, though LDGs are also manifested in marine

337 environments ${ }^{4,22,87}$. Spatio-temporal changes in precipitation, of course, are not directly

338 applicable to marine LDGs, but the same general mechanism of spatio-temporal climate

339 dynamics driving allopatric speciation across real-world seascapes could be responsible for

$340 \mathrm{LDGs}^{22}$.

341 Our results may also depend on the relatively coarse spatial grain of the simulations

$342\left(1^{\circ} \times 1^{\circ}\right)$, as grain is known to affect species richness patterns ${ }^{88,89,90,91}$. The resolution of our

343 simulations was constrained by the spatial resolution of climate data available: analyses at

344 finer scales may produce different results. For example, we did not examine the effect of

345 topography-driven climate heterogeneity on biodiversity patterns in mountainous regions.

346 Topographic, and therefore climatic, heterogeneity could further augment LDGs by

347 prompting allopatric speciation ${ }^{29}$. This mechanism, however, is unlikely to contribute

348 significantly to global-scale LDG patterns, because topographic heterogeneity is not higher at

349 low latitudes (Fig. S56); an argument, of course, which assumes that diversity is promoted in

350 the same way by topographic heterogeneity across latitudes ( see $^{92}$ ). Regardless, the 'climatic

351 flattening' of mountains caused by the coarse resolution of our climate model data does not

352 affect interpretation of the importance of climate parameters in driving evolutionary

353 processes (i.e., precipitation being responsible for low-latitude speciation), since patterns of

354 heterogeneity in temperature and precipitation are likely to be affected similarly ${ }^{93}$. 

climate data. The climate data we have used represent a full, transient global climate trajectory from the last interglacial period to present. No other general circulation model outputs of this nature (i.e., temporally continuous) are available to our knowledge, nor is the fully-coupled HadCM3 model we have used available for earlier time periods. We eagerly await the availability of data on transient changes in climate deeper into the Pleistocene and Pliocene in order to make additional, crucial assessments of the relative roles of Pleistocene and pre-Pleistocene climate dynamics in generating biological diversity. variability as an underlying mechanism for LDGs, proposed previously in landmark papers ${ }^{28}$, ${ }^{29}$. Using virtual-world simulations with minimal biological assumptions, we were able to replicate the shape and strength of the diversity gradient at global and continental scales. Hence, we show that the spatial implications of climate change across real-world landscapes can drive the creation of LDGs, under the assumption of static ecological niches. In this sense, a small number of simple geographic and biological processes are sufficient to explain, either alone or in tandem with others, the generation and maintenance of a major spatial pattern of biodiversity.

\section{Methods}

\section{Simulations}

We used a cellular automaton algorithm to simulate patterns of speciation, extinction, and dispersal in response to a dynamic climate over the last 120,000 years (Table 1). The framework builds on previous models ${ }^{28,94,95}$ to simulate the interaction of virtual species with spatio-temporal variation in temperature and precipitation. Simulations were initiated 
under interglacial climatic conditions (Eemian, Late Pleistocene, $120 \mathrm{Ka}$ ago) and run forward in time to the present-day using current continental configurations (Fig. 4). Each initial lineage had the potential to go extinct or speciate. Speciation and extinction were driven by changing climatic conditions: speciation occurred when ranges were fragmented via isolation of suitable areas for at least 10,000 years (see justification below), and extinction occurred when all occupied suitable areas were eliminated, and the species was unsuccessful at colonizing newly-suitable regions. The simulation protocol is outlined in the Supplementary Materials and is illustrated in Fig. 1. Soft code for the simulation is provided in Appendix 1.

A virtual species began the simulation at a site of origin chosen randomly from within terrestrial areas globally, which were divided into $1^{\circ} \times 1^{\circ}$ grid cells $(15,254$ cells total $)$. Cell occupation was controlled by species' abiotic tolerances and dispersal ability in relation to climatic conditions manifested in the cell. The temperature and precipitation values of the

393 initial starting cell defined the centre of that species' niche. We applied symmetrical

394 deviations to this point ${ }^{28,94}$ based on two niche breadths (narrow and broad), corresponding to temperature and precipitation tolerances of $21^{\circ} \mathrm{C}$ and $31^{\circ} \mathrm{C}$ and $4 \mathrm{~mm} /$ day and $7 \mathrm{~mm} /$ day, respectively. These niche breadths were derived from empirical temperature and precipitation tolerances of agriculturally-important plant species $^{96}$, in which $\sim 18 \%$ of species were characterised as having 'tropical' distributions. Narrow and broad niche breadths were

399 defined using the lower and upper quartiles, respectively, of niche breadths in this database $\mathrm{e}^{94}$.

400 Representation of species' tolerances (fundamental ecological niches) was limited by the

401 climatic combinations existing at a given time step, referred to as the existing niche ${ }^{97}$.

402 Existing niches were further limited by species' ability to access suitable cells, determined by 403 assigned dispersal ability and initial distributions. Seasonality was considered indirectly in 
the model because maximum and minimum temperatures differ more markedly at higher latitudes ${ }^{98}$.

Each species was assigned a dispersal function, reflecting its ability to search outside of its present range for habitable cells. Dispersal in the simulation was stochastic, and represented the process of exploration, with possible colonization and range expansion; this 409 definition therefore differs from other definitions of dispersal at local scales (e.g., movements 410 of individuals). We considered two levels of dispersal ability, both defined by exponential 411 decay curves of the probability that a species will disperse a certain number of cells. From a 412 given occupied cell, a species was allowed to search, at maximum, four (good dispersers) or 413 two (poor dispersers) cells in a single simulation step, corresponding to distances of $>400 \mathrm{~km}$ 414 and >200 km, respectively. Species searched for suitable cells simultaneously from all cells currently occupied, and each cell was assigned a different probability of dispersal. If a species was allowed to disperse two or more cells in a given time step, they could jump over unsuitable regions to encounter more spatially remote but suitable cells elsewhere. As such, 418 dispersal could occur at least occasionally between continents. Dispersal values are provided 419 in Table S5, based loosely on known dispersal abilities of empirically-derived seed-dispersal capacities in plants ${ }^{99,100}$. Considerable uncertainty remains regarding the distances to which

421 plants are able to disperse and colonize areas ${ }^{100}$. However, restricting most dispersal events to one ( $100 \mathrm{~km}$ in size) or no cells per time step (100 years) was considered a close match to reality, since, for example, models for invasive tree species in South Africa estimate rates of up to $\sim 700 \mathrm{~m} \cdot \mathrm{yr}^{-1} 101$.

Climatic attributes of individual cells fluctuated through time and resulted in conditions that were favorable or unfavorable for a species at a given time depending on whether the cell value fell within the species' climatic niche. Dynamic climate change trajectories over the last $120 \mathrm{Ka}$ were derived from transient climate simulations using state- 
of-the-art, coupled atmosphere-ocean-vegetation models (HadCM3) developed at the Hadley

430 Centre $^{102}$. Three climate parameters constrained species' tolerances: mean monthly maximum

431 temperature, mean monthly minimum temperature, and mean monthly maximum

432 precipitation, under three scenarios related to ice sheets (discussed below). Minimum

433 monthly precipitation is zero for nearly all localities on Earth and was therefore not included

434 (precipitation, of course, cannot fall below zero). We also considered simulations when species' tolerances were constrained using mean annual precipitation and mean annual temperature. Resulting patterns were broadly equivalent to simulations using maximum and minimum climate layers, and therefore we focus here only on the latter.

The HadCM3 climate model was originally developed at the UK Met Office and used in previous IPCC future climate projections. The version used here has been described and evaluated by comparison with observational datasets ${ }^{102}$. Simulations were run at 62 time

441 slices, covering the last $120 \mathrm{Ka}$, at a frequency of every 1000 years back to $22 \mathrm{Ka}$, every 2000 years back to $80 \mathrm{Ka}$, and every 4000 years back to $120 \mathrm{Ka}$. The climate model was driven with appropriate boundary conditions for orbital configuration, greenhouse gases derived from ice core records, and ice-sheet volume and topography/bathymetry derived from sea level reconstructions (method described in Eriksson et al. ${ }^{103}$ ). The climate models, which include interactive vegetation, are described in Singarayer et al ${ }^{104}$ and Davies-Barnard et $\mathrm{al}^{105}$. For the purposes of this study, relevant climate outputs were downscaled from $2.50 \mathrm{x}$ $3.75^{\circ}$ horizontal resolution to $1^{\circ}$ resolution using bicubic interpolation, using the climate anomaly method so that predicted modelled changes in climate were added (or using the ratio

450 for precipitation) to an observed present day climatology $y^{62}$. This method removes any systematic bias from the climate model. These data were then interpolated linearly to 100 452 year time steps, resulting in 1201 equal-duration time slices for each of three climatic layers 453 used in the simulation. 
(2) ice sheet present; and (3) varying probability of ice. In the ice sheet model, each cell was assigned a certain percent coverage of ice (from 0 to $100 \%$ ). In the 'probability of ice' scenario, the percent coverage value was used as a probability of elimination of the populations occupying that cell. In the 'ice sheet present' scenario, a cell that became covered in any percentage of ice resulted in complete elimination of the populations living there. The

460 ice sheet extent was derived from Davies-Barnard et al. ${ }^{105}$, and was calculated based on the Ice $5 \mathrm{G}$ ice sheet reconstruction ${ }^{106}$. Ice sheet extent information is available in 1000-year intervals from 26-21 Ka and thereafter every 500 years to present. Reconstruction of ice sheet extent for older periods is difficult, because older evidence has been eroded by ice during the glacial maximum. Consequently, we approximated ice sheet extent prior to $26 \mathrm{Ka}$ by matching sea level. For instance, observed sea level depression is estimated to be $50 \mathrm{~m}$ at $75 \mathrm{Ka}$ and also at $12 \mathrm{Ka}$. Hence, we allocated the same ice mask for both periods. These ice masks were then interpolated linearly in both space and time to produce maps every 100 years. Ice sheet extent and mean global value for each time step and parameter over the last $120 \mathrm{Ka}$ are provided in Fig. S57. In all simulations, species occupied immediately any suitable cell that they encountered via dispersal, and remained there until the cell became unsuitable due to climate change. This process imitates natural range dynamics of species, which are thought often to 473 begin small ${ }^{107,108}$. Climate change modified distributions of suitable cells uniquely for each 474 species (i.e., dependent on niche dimensions); species tracked suitable cells through these 475 changes as a function of their dispersal ability. One consequence of climate change was the 476 fragmentation of suitable areas, resulting in either newly-isolated populations or elimination 477 of all occupied suitable areas (Fig. 1). The former resulted in speciation if populations were 478 isolated for a sufficient length of time (see below), whereas the latter resulted in extinction. 
479 Figure 1 provides a schematic of how this process works within our simulation framework.

480 Simulations also produced a complete phylogeny from each individual starting lineage; these 481 data are stored, although not used in the current analysis.

Minimum isolation time for speciation to occur was set arbitrarily at 10,000 years. In nature, of course, speciation may take longer ${ }^{109}$ or shorter $^{110,111}$ than 10,000 years, but this

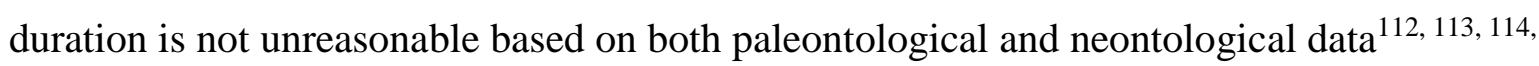
115. Importantly, we chose a time-to-speciation that was proportional to the time steps available in the climate dataset to generate appreciable numbers of speciation events at the scale of climate change steps. The climate model used here represents a full, transitory global climate trajectory from the last interglacial period to present - no other general circulation model outputs of this nature (i.e., temporally 'continuous') are available to our knowledge, nor is the HadCM3 model available for earlier periods. Importantly, other parallel simulations by Rangel et $\mathrm{al}^{29}$ found that time-to-speciation did not have significant effects on model results.

Following Qiao et $\mathrm{al}^{94}$, daughter species have identical niches and dispersal abilities as their parent species. That is, no evolution of niche or dispersal traits occurs through time, which invokes the fewest assumptions regarding demographic processes, genetic/phenotypic plasticity, and evolvability during speciation. Niche evolution was purposefully avoided to isolate the effects of the spatial distribution of climate through time on the latitudinal structure of diversity. In natural systems, niche breadth and dispersal ability will vary among species; how this variation may affect macroecological patterns is not treated here, but has

500 been discussed elsewhere ${ }^{29}$. Populations that separated and re-merged in less than 10,000 501 years were not regarded as distinct species. Once produced, daughter species immediately 502 began to search and occupy all suitable cells within their dispersal reach, and were not 503 prohibited from cell occupation by presence of another species. In this sense, biotic 
interactions were not included in our simulations: species were not excluded from suitable areas based on presence of other species, nor did presence of other species affect extinction probability. Again, excluding the effects of biotic interactions was deliberate to reduce confounding variables, whilst examining whether biodiversity patterns can be explained by a

508 few simple processes.

509 Extinction occurred when all occupied suitable habitat (i.e., cells) for a species 510 disappeared and the species was unsuccessful at colonizing new areas. We applied no 511 specific demographic model or inferred minimum population survivorship threshold ${ }^{94}$, such

512 as might derive from the existence of Allee effects. A strict extinction criterion was used

513 because it also invoked the fewest assumptions, and because the relatively coarse spatial

514 resolution of the simulation (i.e., $110 \mathrm{~km} \times 110 \mathrm{~km}$ at the Equator) was likely broad with respect to individual life histories.

In all, 2000 initial species locations were chosen randomly (Fig. S58) and tested under

517 all combinations of niche breadth (narrow and broad), dispersal ability (poor and good), and

518 climate scenario (no ice, ice, and probability of ice), resulting in 24,000 unique virtual

519 species simulations. Each of these 24,000 simulations started as a point in space: at the end of 520 each simulation, the final spatial pattern of diversity was quantified, including all extant species that had evolved over the course of the simulation. We additionally documented the location and frequency of the speciation and extinction events that generated this biodiversity. Although we considered 2000 initial seed points for each ecological combination, patterns of diversity were analyzed using standard numbers of seed points in each latitudinal band (discussed below), such that no diversity gradient existed at the beginning of each analysis (Fig. S59). 
530 from $120 \mathrm{Ka}$ using the 'no ice' climate model. Species could occupy suitable cells based on

531 their niches and dispersal abilities, but climate remained static over the course of the $120 \mathrm{Kyr}$

532 simulation (note that temperature seasonality was still considered implicitly, since there is

533 greater disparity between maximum and minimum temperatures at high latitudes). No

534 speciation or extinction occurred in the simulations, since these processes are driven by spatio-temporal dynamics of climate change. These 'no climate change' simulations served as a null model against which to compare patterns resulting from spatio-temporal dynamics

537 of climate, and thus relied on the same initial seeds and parameters as the original

538 simulations.

539

Patterns of diversity by latitude

541 Global terrestrial areas were seeded randomly with incipient species, such that some

542 latitudes differed in numbers of species simulated, reflecting different land areas. To account

543 for this uneven initial starting diversity, we drew randomly one simulation from each $1^{\circ}$

544 latitudinal band for a series of 100 bootstrap replicates. This process standardized initial

545 diversity by latitude (i.e., one initial seed or 'species'), which was necessary since we did not

546 wish to a priori generate latitudinal diversity gradients (Fig. S59). From each bootstrap

547 replicate, we quantified the number of species at each degree of latitude at the end of the

548 simulations, a process repeated for every dispersal $\times$ niche $\times$ climate scenario combination.

549 Mean and median latitudinal diversity and associated 95\% confidence intervals from the 100

550 bootstrap replicates are shown in Fig. 2 and Figs. S1-6. Note that variance among bootstrap

551 samples may depend on longitudinal extent of each latitudinal band (Fig. S58). We also

552 present results scaled to unit land area available per latitudinal band (Figs. S7-12). 
Regional biodiversity patterns were assessed using the bootstrap framework described

554 above for Eurafrica (Europe \& Africa), Australasia (Australia and Asia), and the Americas

555 for both raw diversity (Figs. S13-15) and diversity scaled to area (Figs. S16-18). These

556 analyses considered only 139 latitudinal bands (from $-55^{\circ}$ to $83^{\circ}$ ), removing highest latitudes

557 because land area was minimal and lacked initial species seeds.

558

Speciation and extinction by latitude

Using the bootstrap framework described above, we quantified speciation and

561 extinction rates by latitude by pulling randomly one simulation from each $1^{\circ}$ latitudinal band

562 for a series of 100 bootstrap replicates. Two methods were used to determine where speciation events occurred: (1) cells were included if they were within the distribution of the two diverging populations at the time of speciation; or (2) each unoccupied, empty cell (i.e., the barrier) between diverging populations was included. For the latter method, we calculated a convex hull around the distributional areas of the diverging populations at the time of speciation. The cells within this convex hull, but not within the distribution of either population, were counted as the area of speciation. The two methods produced almost identical results, so we present results for only the latter. We document where an extinction event occurred using the entire historical distribution of the species, as the distribution prior to extinction was often represented by only one cell. Using this framework, we generated

572 characterizations of speciation (Fig. 3; Figs. S22-24) and extinction (Fig. 3; Figs. S28-30) per latitude for the different niche $\times$ dispersal $\times$ climate scenario combinations, and also scaling both metrics to per-unit area in each latitudinal band (Figs. S25-27 and S31-33). 
basis. Maps were generated by selecting one simulation per latitudinal band. Numbers of

events (presence of species, extinction or speciation events) were then tabulated for each cell

580 globally (Fig. 4; Fig. S35). This process was repeated 100 times, and the results summed across replicates. Speciation and extinction were considered using the same framework outlined above (Fig. S35).

\section{Dispersal dynamics}

We documented the frequency of shifts from temperate to tropical biomes and vice versa to examine the role of dispersal in generating diversity gradients. Tropical and temperate regions were delineated as those regions having mean annual temperatures greater and less than $18^{\circ} \mathrm{C}$, respectively ${ }^{116,117}$ (Fig. S60). In the first two analyses, we selected randomly 10 simulations per biome per replicate over a series of 100 bootstrap replicates. We counted either (1) the number of initial incipient lineages with any species that originated in

591 the tropics and dispersed to temperate regions and vice versa. Using this method, a maximum

592 of 10 dispersal events could be counted for species dispersing from temperate to tropical regions or vice versa per bootstrap (Figs. S36-38); or (2) the total number of any species that dispersed from the tropics to temperate regions and vice versa from these initial incipient lineages (Fig. 5; Figs. S39-41). Higher out-of-the-tropics expansion becomes likely if

596 diversity is higher in the tropics ${ }^{45}$, so we standardized the number of potential dispersal 597 events from tropical to temperate regions and vice versa in a third analysis. In this method, 598 we selected randomly 1000 species that were 'born' (i.e., simulations initiated) in tropical 599 regions and counted the proportion of those species that dispersed from the tropics to 600 temperate regions in a series of 100 bootstrap replicates; this process was then repeated for 601 species born in, and selected randomly from, temperate regions (Figs. S42-44). 

as areas within $+/-23.5^{\circ}$ latitude, regardless of climate. Dispersal analyses were repeated using this latitudinal definition of biomes. Results, however, were similar and therefore are not shown.

Climate dynamics

The spatio-temporal dynamics of climate change drove speciation and extinction in

609 the simulation framework. We quantified the relative contributions of the three climatic

610 parameters (maximum precipitation, maximum temperature, and minimum temperature) to

611 speciation and extinction across latitudes. For speciation, we determined the relative

612 contribution of each climate parameter to unsuitable regions (i.e., the barriers) between

613 populations, which prompted allopatric speciation. Barriers were identified by generating a

614 convex hull around the distributional area of diverging populations at the time of speciation

615 using the centre of each cell: cell centres within this convex hull, but not within the

616 distribution of either population, were considered part of the 'barrier' that promoted

617 speciation. This region, however, could be unoccupied because of dispersal constraints, or

618 because one or more climatic parameters were outside the tolerance limits for a given species.

619 We analysed only the latter scenario, removing from consideration any pixel that was suitable

620 but unoccupied due to dispersal limitations. For each 'barrier' pixel in a speciation event, we

621 documented unsuitability with respect to the three climatic parameters, indicating the pixel

622 was outside the species' niche and therefore causing range fragmentation and ultimately

623 speciation. This process was repeated for 1000 random speciation events every 5000 years

624 (for a total of 50 time steps). Each pixel associated with speciation was assigned three values

625 representing the proportion of speciation events in that pixel caused by: (i) unsuitability in

626 maximum precipitation, (ii) unsuitability in maximum temperature, and (iii) unsuitability in 
627 minimum temperature. Note that these values need not sum to unity, since a pixel could be

628

629

630

631

632

633

634

635

636

637

638

639

640

641

642

643

644

645

646

647

648

649

650

651

unsuitable in multiple climate dimensions. Mean proportions were calculated for each climate variable in each latitudinal band and time step, for all niche breadth and dispersal combinations (Fig. 6; Figs. S45-S47).

The same framework was used to assess the relative contributions of the three climate variables to extinction across latitudes. We considered the climate conditions in the time step in which the species disappeared (i.e., no longer occupied any pixels), but determined which of the climate variables caused extinction by looking to the pixels occupied in the time step immediately preceding the extinction event. In each of these previously-occupied pixels, we assigned values representing the proportion of extinction events in that pixel caused by: (i) unsuitability in maximum precipitation, (ii) unsuitability in maximum temperature, and (iii) unsuitability in minimum temperature. Mean proportions were calculated for each climate variable in each latitudinal band and time step analysed (Fig. 6; Fig. S48-S50). As with speciation, we considered the effect of niche breadth and dispersal combinations.

In addition to assessing the relative contribution of the three climate variables to speciation and extinction, we quantified spatio-temporal variability of each climatic parameter over the last $120 \mathrm{Ka}$. Variability was measured as the standard deviation of conditions in a particular cell across the $120 \mathrm{Ka}$ (Fig. S51) using the 'no ice' climate scenario, since the other scenarios use the ice sheet only as a 'mask' to remove occupied cells.

\section{Comparison with empirical patterns}

We compared simulation results to empirical patterns of global terrestrial diversity for birds, mammals, and amphibians, because diversity data for these taxa were readily available worldwide, which was not the case for plants (i.e., no global database was available to us). 
652 Diversity maps were derived from BiodiversityMapping.org ${ }^{118,119}$, which in turn obtained 653 data from BirdLife International and NatureServe (birds) and the IUCN (mammals and 654 amphibians; http://www.iucnredlist.org). We coarsened maps from $10 \mathrm{~km}$ x $10 \mathrm{~km}$ resolution 655 to match the $1^{\circ}$ resolution of our simulations using bilinear interpolation.

656 We standardized both empirical and simulated diversity maps on a scale from 0 to 1

657 by dividing by the highest diversity value in each. This step allowed us to compare the shape 658 of curves and relative distribution of diversity, rather than the absolute magnitude of species 659 counts, since total number of species differs amongst groups (for example, $\sim 10,000$ in birds 660 and only $\sim 5,400$ in mammals exist globally) and is arbitrary in our simulations. Using the 661 standardized maps, we quantified similarity between our simulations and empirical diversity 662 patterns in four ways: (1) We calculated correlations (Pearson's $r$ ) between simulated diversity and empirical diversity on a cell-by-cell basis (Table S1). We compared the level of correlation to null expectations, generated by reshuffling the simulated diversity values within each $1^{\circ}$ latitudinal band and quantifying correlations between these reshuffled maps and the empirical maps. This process was repeated 100 times to generate a count of instances 667 in which the strength of correlation was lower using the reshuffled maps (Fig. S52; Table 668 S2). Note that the amphibian diversity map lacked data in some regions (Fig. 4), which were excluded from analyses. (2) We calculated model residuals by subtracting simulated diversity

670 from empirical diversity to examine regions in which the simulations over- or under-

671 predicted richness (Fig. S53). Analyses were performed using the 'raster' package in $\mathrm{R}^{120}$.

672 We quantified similarity between simulated and empirical latitudinal diversity curves using 673 Fréchet distances (Fig. 2), which measure similarity between two curves while considering 674 the location and ordering of the points along the curves. The metric is often described in 675 terms of a person walking a dog on a leash, with the person walking along one curve and the 676 dog along another ${ }^{121}$. The dog and person are able to vary their speeds, or even stop, but not 
677 go backwards. The Fréchet metric is the minimum leash length required to complete the traversal of both curves. We calculated this metric between all pair-wise combinations of simulated diversity curves and empirical diversity curves using the 'Frechet' function in the

680 SimilarityMeasures package for R 3.3.1 ${ }^{122}$ (Table S3). We additionally used Kolmogorov-

681 Smirnov two-sample tests to compare similarities between all pair-wise combinations of

682 simulated diversity curves and empirical diversity curves. These tests suggested statisticallysignificant differences among all diversity gradients $(p<0.0001)$, including among empirical clades (e.g., between birds and mammals), and thus we do not dwell further on these results. Finally, (4) we compared geographic range-size frequency distributions (Fig. S54). We assessed the similarity of the range-size frequency distributions for virtual species at the end of the simulations with range-size frequency distributions for species of birds, mammals, and amphibians. Each species' distribution was represented as a polygon in a vector GIS shapefile. Shapefiles for each species in the three groups were rasterized from the IUCN (mammals and amphibians) and BirdLifeInternational (birds) to $1^{\circ}$ resolution. A species was considered to occupy a given cell if any part of that species' range intersected the cell; the total number of occupied cells was then summed across the species' range. We repeated this process for each virtual, bird, mammal, and amphibian species. Range-size frequency distributions for birds, mammals, and amphibians were then compared statistically to simulated range-size frequency distributions by niche $\times$ dispersal $\times$ climate scenario combination using Kolmogorov-Smirnov two-sample tests in R 3.1.1. Given our ability to

697 generate many more species than are often observed in empirical groups (405,480 in total), 69810,000 simulated species were selected randomly from each niche $\times$ dispersal $\times$ climate 699 scenario combination for comparison to the empirical patterns, in each of a series of 100 700 replicates. 
703 We thank Robert Colwell (U Connecticut) and Fabien Condamine (CNRS) for comments that greatly improved our contribution. We are indebted to Dan Hill (Leeds, UK), Paul Wignall (Leeds, UK), and Roger Benson (Oxford, UK) for thoughtful discussions that informed this manuscript. H.Q. was supported by the National Key Research and Development Project of China (2017YFC1200603), Natural Science Foundation of China (31772432). EES acknowledges funding from an EAR NSF Postdoctoral Fellowship and Leverhulme Grant no. DGR01020. CEM acknowledges funding from NSF \#1601878. All data are available via Dryad: doi:10.5061/dryad.m6h850q. Note that the soft code for the simulations is provided in 711 Appendix 1 and freely available for use.

\section{Author contributions}

714 EES designed the study, EES and HQ performed the analyses, J. Singarayer and PV provided climate data and analysis, EES and HQ analyzed the results. EES wrote the first draft of the manuscript, and all authors (CEM, ATP, JS, J. Singarayer, PV, HQ) contributed to revisions.

The authors declare no competing interests.

Supplementary Materials

Supplementary Simulation Protocol

722 Supplementary Tables (S1-S5)

723 Supplementary Figures (S1-S60)

Table and Figures

Table 1. Model parameters and their permutations used in the simulations. A detailed explanation of all scenarios and parameters are provided in the main text methods.

Figure 1. Schematic of the process of speciation and extinction in the simulation framework. Speciation occurs in response to range fragmentation driven by climate change. Speciation will occur only if populations remain isolated for at least 10,000 years. Extinction is also climatically mediated, and occurs when a species is no longer able to occupy suitable cells or suitable cells do not exist. See 'Simulation Protocol' in the Supplementary Materials for further details.

Figure 2. Standardized mean number of species per $1^{\circ}$ latitudinal band with standard error bars. Simulated results are shown only for the 'no ice' climate scenario for all dispersal and niche combinations, and for species with narrow niches and poor dispersal abilities. Results were similar for other climate scenarios, albeit even closer matches to empirical diversity curves, shown in Figs. S1-6 and scaled to area in Figs. S7-12. 
Figure 3. Mean speciation (top) and extinction (bottom) rate per latitudinal band with associated $95 \%$ confidence intervals across 100 bootstrap replicates. Values include all speciation and extinction events that occurred over the 120-kyr simulation for species with narrow niches aggregated across both dispersal abilities. Three climate scenarios are shown, which are explained in detail in the Methods. Parallel graphics with speciation and extinction rates scaled to area are given as Figs. S25-27 and S31-33.

Figure 4. Distribution of bird, mammal, and amphibian species, as well as virtual species with narrow niches and poor dispersal abilities in the present day. Simulated results are shown only for the 'no ice sheet' climate scenario, but patterns do not differ significantly for other climate scenarios. Extant vertebrate data were derived from BiodiversityMapping.org ${ }^{118,119}$. Latitudinal diversity curves were generated by quantifying mean diversity in each $1^{\circ}$ latitudinal band with associated standard deviations. Note the variability among the three empirical diversity maps and curves.

Figure 5. Frequency of shifts from temperate to tropical biomes and vice versa by virtual species across 100 bootstrap replicates for all dispersal and niche combinations (for other dispersal and niche combinations, see Figs. S36-44). Results show the total number of species that dispersed from the tropics to temperate regions and vice versa from initial incipient lineages. Three climate scenarios are shown, which are explained in detail in the Methods.

Figure 6. Mean contribution of climate parameters to speciation (top) and extinction (bottom) in each $1^{\circ}$ latitudinal band with associated $95 \%$ confidence intervals that reflect temporal variation during the simulation. Each pixel associated with speciation and extinction was assigned three values representing the proportion of events in that pixel caused by (i) unsuitability in maximum precipitation, (ii) unsuitability in maximum temperature, and/or (iii) unsuitability in minimum temperature. Note that these values need not sum to unity, since a pixel could be unsuitable in all three climate dimensions. Mean proportions were calculated for each climate variable in each latitudinal band and in each time step. Results are shown for all dispersal and niche combinations. No confidence intervals are shown for extinction, as variability was so high that it obscured mean patterns. Max precip = maximum precipitation; $\max$ temp $=$ maximum temperature , and $\min$ temp $=$ minimum temperature. Other niche and dispersal scenarios are shown in Figs. S45-50. 
822

823

824

825

826

827

828

829

830

831

832

833

834

835

836

837

838

839

840

841

842

843

844

\section{References}

1. Fine PV. Ecological and evolutionary drivers of geographic variation in species diversity. Annu Rev Ecol Evol Syst 46, 369-392 (2015).

2. Hillebrand H. On the generality of the latitudinal diversity gradient. The American Naturalist 163, 192-211 (2004).

3. Willig MR, Kaufman DM, Stevens RD. Latitudinal gradients of biodiversity: pattern, process, scale, and synthesis. Annu Rev Ecol Evol Syst 34, 273-309 (2003).

4. Valentine JW, Jablonski D. A twofold role for global energy gradients in marine biodiversity trends. J Biogeogr 42, 997-1005 (2015).

5. Roy K, Jablonski D, Valentine JW. Dissecting latitudinal diversity gradients: functional groups and clades of marine bivalves. Proceedings of the Royal Society of London B 267, 293-299 (2006).

6. Kerkhoff AJ, Moriarty PE, Weiser MD. The latitudinal species richness gradient in New World woody angiosperms is consistent with the tropical conservatism hypothesis. Proceedings of the National Academy of Sciences 111, 8125-8130 (2014).

7. Qian H, Ricklefs RE. A latitudinal gradient in large-scale beta diversity for vascular plants in North America. Ecol Lett 10, 737-744 (2007).

8. Cardillo M. Latitude and rates of diversification in birds and butterflies. Proceedings of the Royal Society of London B 266, 1221-1225 (1999).

9. Buckley LB, et al. Phylogeny, niche conservatism and the latitudinal diversity gradient in mammals. Proceedings of the Royal Society of London B rspb20100179, (2010).

10. Rolland J, Condamine FL, Jiguet F, Morlon H. Faster speciation and reduced extinction in the tropics contribute to the mammalian latitudinal diversity gradient. PLoS Biol 12, e1001775 (2014).

11. Powell MG. Latitudinal diversity gradients for brachiopod genera during late Palaeozoic time: links between climate, biogeography and evolutionary rates. Glob Ecol Biogeogr 16, 519-528 (2007).

12. Mannion PD, Upchurch P, Benson RBJ, Goswami A. The latitudinal biodiversity gradient through deep time. Trends Ecol Evol 29, 42-50 (2014).

13. Crame JA. Taxonomic diversity gradients through geological time. Divers Distrib 7, 175-189 (2011).

14. Brown JH. Why are there so many species in the tropics? J Biogeogr 41, 8-22 (2014).

15. Mittelbach GG, et al. Evolution and the latitudinal diversity gradient: speciation, extinction and biogeography. Ecol Lett 10, 315-331 (2007). 
16. Pianka ER. Latitudinal gradients in species diversity: a review of concepts. Am Nat 100, 33-46 (1966).

17. Stebbins GL. Flowering Plants: Evolution above the Species Level. The Belknap Press of Harvard University Press (1974).

18. Chown SL, Gaston KJ. Areas, cradles and museums: the latitudinal gradient in species richness. Trends in Ecology \& Evolution 15, 311-315 (2000).

19. Jablonski D, Roy K, Valentine JW. Out of the tropics: evolutionary dynamics of the latitudinal diversity gradient. Science 314, 102-106 (2006).

20. Gaston KJ, Blackburn TM. The tropics as a museum of biological diversity: an analysis of the New World avifauna. Proceedings of the Royal Society B 263, 63-68 (1996).

21. Arita HT, Vázquez-Domínguez E. The tropics: cradle, museum or casino? A dynamic null model for latitudinal gradients of species diversity. Ecol Lett 11, 653-663 (2008).

22. Roy K, Goldberg EE. Origination, extinction, and dispersal: integrative models for understanding present-day diversity gradients. The American Naturalist 170, S71-S85 (2007).

23. Wiens JJ, Graham CH, Moen DS, Smith SA, Reeder TW. Evolutionary and ecological causes of the latitudinal diversity gradient in hylid frogs: treefrog trees unearth the roots of high tropical diversity. The American Naturalist 168, 579-596 (2006).

24. Pyron RA, Wiens JJ. Large-scale phylogenetic analyses reveal the causes of high tropical amphibian diversity. Proceedings of the Royal Society of London B 280, 20131622 (2013).

25. Jablonski D, Huang S, Roy K, Valentine JW. Shaping the latitudinal diversity gradient: new perspectives from a synthesis of paleobiology and biogeography. The American Naturalist 189, 1-12 (2017).

26. Tittensor DP, Worm B. A neutral-metabolic theory of latitudinal biodiversity. Glob Ecol Biogeogr 25, 630-641 (2016).

27. Dynesius M, Jansson R. Evolutionary consequences of changes in species' geographical distribution driven by Milankovitch climate oscillations. Proceedings of the National Academy of Sciences of the USA 97, 9115-9120 (2000).

28. Rangel TFL, Diniz-Filho JAF, Colwell RK. Species richness and evolutionary niche dynamics: a spatial pattern-oriented simulation experiment. The American Naturalist 170, 602-616 (2007).

29. Rangel TFL, et al. Modeling the ecology and evolution of biodiversity: Biogeographical cradles, museums, and graves. Science 361, eaar5452 (2018). 
30. Jackson ST, Overpeck JT. Responses of plant populations and communities to environmental changes of the late Quaternary. Paleobiology 26, 194-220 (2000).

31. Haffer J, Prance GT. Climatic forcing of evolution in Amazonia during the Cenozoic: on the refuge theory of biotic differentiation. Amazoniana 16, 579-607 (2001).

900

901

32. Wiens JJ, Donoghue MJ. Historical biogeography, ecology and species richness. Trends in Ecology and Evolution 19, 639-644 (2004).

33. Pyron RA, Costa GC, Patten MA, Burbrink FT. Phylogenetic niche conservatism and the evolutionary basis of ecological speciation. Biological Reviews 90, 1248-1262 (2015).

34. Strubbe D, Beauchard O, Matthysen E. Niche conservatism among non-native vertebrates in Europe and North America. Ecography 38, 321-329 (2015).

35. Peterson AT, Soberón J, Sánchez-Cordero V. Conservatism of ecological niches in evolutionary time. Science 285, 1265-1267 (1999).

36. Saupe E, et al. Macroevolutionary consequences of profound climate change on niche evolution in marine molluscs over the past three million years. Proceedings of the Royal Society of London B 281, 20141995 (2014).

37. Barnes R, Clark AT. Sixty-five million years of change in temperature and topography explain evolutionary history in eastern North American plethodontid salamanders. The American Naturalist 190, E1-E12 (2017).

38. Rahbek C, Gotelli NJ, Colwell RK, Entsminger GL, Rangel TFL, Graves GR. Predicting continental-scale patterns of bird species richness with spatially explicit models. Proceedings of the Royal Society of London B: Biological Sciences 274, 165174 (2007).

39. Cowling SA, Maslin MA, Sykes MT. Paleovegetation simulations of lowland Amazonia and implications for neotropical allopatry and speciation. QuRes 55, 140149 (2001).

40. Gotelli NJ, et al. Patterns and causes of species richness: a general simulation model for macroecology. Ecol Lett 12, 873-886 (2009).

41. Connolly SR, Keith SA, Colwell RK, Rahbek C. Process, mechanism, and modeling in macroecology. Trends Ecol Evol 32, 835-844 (2017).

42. Romdal TS, Araújo MB, Rahbek C. Life on a tropical planet: niche conservatism and the global diversity gradient. Glob Ecol Biogeogr 22, 344-350 (2013).

43. Cabral JS, Valente L, Hartig F. Mechanistic simulation models in macroecology and biogeography: state-of-art and prospects. Ecography 40, 267-280 (2017). 
44. Jablonski D, et al. Out of the tropics, but how? Fossils, bridge species, and thermal ranges in the dynamics of the marine latitudinal diversity gradient. Proceedings of the National Academy of Sciences 110, 10487-10494 (2013).

45. Tomašových A, Jablonski D. Decoupling of latitudinal gradients in species and genus geographic range size: a signature of clade range expansion. Glob Ecol Biogeogr 26, 288-303 (2017).

46. Anderson S. Geographic ranges of North American terrestrial mammals. Am Mus Novit 2629, 1-15 (1977).

47. Brown JH, Stevens GC, Kaufman DM. The geographic range: size, shape, boundaries, and internal structure. Annual Review of Ecology, Evolution and Systematics 27, 597-623 (1996).

48. Gaston KJ. Species-range-size distributions: patterns, mechanisms and implications. Trends in Ecology and Evolution 11, 197-201 (1996).

49. Crane PR, Lidgard S. Angiosperm diversification and paleolatitudinal gradients in Cretaceous floristic diversity. Science 246, 675-678 (1989).

50. Jansson R, Rodríguez-Castañeda G, Harding LE. What can multiple phylogenies say about the latitudinal diversity gradient? A new look at the tropical conservatism, out of the tropics, and diversification rate hypotheses. Evolution 67, 1741-1755 (2013).

51. MacArthur RH, Wilson EO. The Theory Of Island Biogeography. Princeton University Press (1967).

52. Whittaker RJ, Ferna' ndez-Palacios JM. Island Biogeography. Oxford University Press (2007).

53. Baselga A, Lobo JM, Svenning JC, Aragón P, Araújo MB. Dispersal ability modulates the strength of the latitudinal richness gradient in European beetles. Glob Ecol Biogeogr 21, 1106-1113 (2012).

54. Davies TJ, et al. Phylogenetic trees and the future of mammalian biodiversity. Proceedings of the National Academy of Sciences 105, 11556-11563 (2008).

55. Davies TJ, Buckley LB, Grenyer R, Gittleman JL. The influence of past and present climate on the biogeography of modern mammal diversity. Philosophical Transactions of the Royal Society B 366, 2526-2535 (2011).

56. Sexton JP, Montiel J, Shay JE, Stephens MR, Slatyer RA. Evolution of ecological niche breadth. Annu Rev Ecol Evol Syst 48, 183-206 (2017).

57. Sunday JM, Bates AE, Dulvy NK. Global analysis of thermal tolerance and latitude in ectotherms. Proceedings of the Royal Society of London B 278, 1823-1830 (2012). 
58. Hillman SS, Drewes RC, Hedrick MS, Hancock TV. Physiological vagility: correlations with dispersal and population genetic structure of amphibians. Physiol Biochem Zool 87, 105-112 (2013).

59. Bonetti MF, Wiens JJ. Evolution of climatic niche specialization: a phylogenetic analysis in amphibians. Proceedings of the Royal Society of London B 281, 20133229 (2014).

60. Cheng $\mathrm{H}$, et al. Climate change patterns in Amazonia and biodiversity. Nature Communications 4, 1411 (2013).

61. Wang X, et al. Hydroclimate changes across the Amazon lowlands over the past 45,000 years. Nature 541, 204-207 (2017).

62. New M, Lister D, Hulme M, Makin I. A high-resolution data set of surface climate over global land areas. Clim Res 21, 1-25 (2002).

63. Held IM, Soden BJ. Robust responses of the hydrological cycle to global warming. JCli 19, 5686-5699 (2006).

64. Fischer AG. Latitudinal variations in organic diversity. Evolution 14, 64-81 (1960).

65. Weir JT, Schluter D. The latitudinal gradient in recent speciation and extinction rates of birds and mammals. Science 315, 1574-1576 (2007).

66. Hawkins BA, Diniz-Filho JAF, Jaramillo CA, Soeller SA. Post-Eocene climate change, niche conservatism and the latitudinal diversity gradient of New World birds. J Biogeogr 33, 770-780 (2006).

67. Simpson GG. Species density of North American recent mammals. Syst Zool 13, 5773 (1964).

68. Wallace AR. Tropical nature, and other essays. Macmillan and Company (1878).

69. Vrba E. Ecology in relation to speciation rates: some case histories of MioceneRecent mammal clades. Evol Ecol 1, 283-300 (1987).

70. Vrba ES. Environment and evolution: alternative causes of the temporal distribution of evolutionary events. S Afr J Sci 81, 229-236 (1985).

71. Carmichael MJ, et al. A model-model and data-model comparison for the early Eocene hydrological cycle. CliPa 12, 455-481 (2016).

72. Carmichael MJ, et al. Hydrological and associated biogeochemical consequences of rapid global warming during the Paleocene-Eocene Thermal Maximum. Glob Planet Change 157, 114-138 (2017).

73. Webb T, Bartlein PJ. Global changes during the last 3 million years: climatic controls and biotic responses. Annu Rev Ecol Syst 23, 141-173 (1992). 
74. Caley T, et al. A two-million-year-long hydroclimatic context for hominin evolution in southeastern Africa. Nature 560, 76-79 (2018).

75. Jablonski D. Extinction and the spatial dynamics of biodiversity. Proceedings of the National Academy of Sciences USA 105, 11528-11535 (2008).

76. Powell MG. The latitudinal diversity gradient of brachiopods over the past 530 million years. The Journal of Geology 117, 585-594 (2009).

77. Kiel S, Nielson SN. Quaternary origin of the inverse latitudinal diversity gradient among southern Chilean mollusks. Geo 38, 955-958 (2012).

78. Archibald SB, Bossert WH, Greenwood DR, Farrell BD. Seasonality, the latitudinal gradient of diversity, and Eocene insects. Paleobiology 36, 374-398 (2010).

79. Krug AZ, Jablonski D, Valentine JW, Roy K. Generation of Earth's first-order biodiversity pattern. Astrobiology 9, 113-124 (2009).

80. Jetz W, Fine PV. Global gradients in vertebrate diversity predicted by historical areaproductivity dynamics and contemporary environment. PLoS Biol 10, e1001292 (2012).

81. Schemske DW, Cornell HV, Mittelbach GG, Roy K, Sobel JM. Is there a latitudinal gradient in the importance of biotic interactions? Annu Rev Ecol Syst 40, 245-269 (2009).

82. Ezard TH, Purvis A. Environmental changes define ecological limits to species richness and reveal the mode of macroevolutionary competition. Ecol Lett 19, 899906 (2016).

83. Allen AP, Gillooly JF, Savage VM, Brown JH. Kinetic effects of temperature on rates of genetic divergence and speciation. Proceedings of the National Academy of Sciences USA 103, 9130-9135 (2006).

84. Pigot AL, Tobias JA, Jetz W. Energetic constraints on species coexistence in birds. PLoS Biol 14, e1002407 (2016).

85. Colwell RK, Lees DC. The mid-domain effect: geometric constraints on the geography of species richness. Trends Ecol Evol 15, 70-76 (2000).

86. Romdal TS, Colwell RK, Rahbek C. The influence of band sum area, domain extent, and range sizes on the latitudinal mid-domain effect. Ecology 86, 235-244 (2005).

87. Tittensor DP, et al. Global patterns and predictors of marine biodiversity across taxa. Nature 466, 1098 (2010).

88. Rahbek C, Graves GR. Multiscale assessment of patterns of avian species richness. Proceedings of the National Academy of Sciences 98, 4534-4539 (2001). 
89. Lyons SK, Willig MR. Species richness, latitude, and scale-sensitivity. Ecology 83, 47-58 (2002).

90. Kaspari M, Yuan M, Alonso L. Spatial grain and the causes of regional diversity gradients in ants. Am Nat 161, 459-477 (2003).

91. Lira-Noriega A, Soberón J, Navarro-Sigüenza AG, Nakazawa Y, Peterson AT. Scale dependency of diversity components estimated from primary biodiversity data and distribution maps. Diversity and Distributions 13, 185-195 (2007).

92. Janzen DH. Why mountain passes are higher in the tropics. Am Nat 101, 233-249 (1967).

93. New M, Hulme M, Jones P. Representing twentieth-century space-time climate variability. Part II: Development of 1901-96 monthly grids of terrestrial surface climate. JCli 13, 2217-2238 (2000).

94. Qiao H, Saupe EE, Soberón J, Peterson AT, Myers CE. Impacts of niche breadth and dispersal ability on macroevolutionary patterns. Am Nat 188, 149-162 (2016).

95. Tomašových A, Jablonski D, Berke SK, Krug AZ, Valentine JW. Nonlinear thermal gradients shape broad-scale patterns in geographic range size and can reverse Rapoport's rule. Glob Ecol Biogeogr 24, 157-167 (2015).

96. Hijmans R, Guarino L, Cruz M, Rojas E. Computer tools for spatial analysis of plant genetic resources data: 1. DIVA-GIS. Plant Genetic Resources Newsletter, 15-19 (2001).

97. Jackson ST, Overpeck JT. Responses of plant populations and communities to environmental changes of the late Quaternary. Paleobiology 26, 194-220 (2000).

98. Saupe EE, et al. Non-random latitudinal gradients in range size and niche breadth predicted by spatial patterns of climate. Glob Ecol Biogeogr, (2019).

99. Cain ML, Milligan BG, Strand AE. Long-distance seed dispersal in plant populations. Am J Bot 87, 1217-1227 (2000).

100. Svenning JC, Sandel B. Disequilibrium vegetation dynamics under future climate change. Am J Bot 100, 1266-1286 (2013).

101. Higgins SI, et al. Forecasting plant migration rates: managing uncertainty for risk assessment. J Ecol 91, 341-347 (2003).

102. Valdes PJ, et al. The BRIDGE HadCM3 family of climate models: HadCM3@ Bristol v1.0. Geoscientific Model Development, (2017).

103. Eriksson A, et al. Late Pleistocene climate change and the global expansion of anatomically modern humans. Proceedings of the National Academy of Sciences $\mathbf{1 0 9}$, 16089-16094 (2012). 
104. Singarayer JS, Valdes PJ, Roberts WHG. Ocean dominated expansion and contraction of the late Quaternary tropical rainbelt. Sci Rep 7, 9382 (2017).

105. Davies-Barnard T, Ridgwell A, Singarayer JS, Valdes PJ. Quantifying the influence of the terrestrial biosphere on glacial-interglacial climate dynamics. Climates of the Past 13, 1381 (2017).

106. Peltier WR. Global glacial isostasy and the surface of the ice-age Earth: the ICE-5G (VM2) model and GRACE. Annu Rev Earth Planet Sci 32, 111-149 (2004).

107. Liow LH, Stenseth NC. The rise and fall of species: implications for macroevolutionary and macroecological studies. Proceedings of the Royal Society of London B 274, 2745-2752 (2007).

108. Webb TJ, Gaston KJ. Geographic range size and evolutionary age in birds. Proceedings of the Royal Society of London B 267, 1843-1850 (2000).

109. Avise JC. Pleistocene phylogeographic effects on avian populations and the speciation process. Proceedings of the Royal Society of London B 265, 457-463 (1998).

110. Hendry AP, Nosil P, Rieseberg LH. The speed of ecological speciation. Funct Ecol 21, 455-464 (2007).

111. Lamichhaney S, Han F, Webster MT, Andersson L, Grant BR, Grant PR. Rapid hybrid speciation in Darwin's finches. Science 359, 224-228 (2018).

112. Johnson NK, Cicero C. New mitochondrial DNA data affirm the importance of Pleistocene speciation in North American birds. Evolution 58, 1122-1130 (2004).

113. Knowles L, Alvarado-Serrano DF. Exploring the population genetic consequences of the colonization process with spatio-temporally explicit models: insights from coupled ecological, demographic and genetic models in montane grasshoppers. $\mathrm{Mol}$ Ecol 19, 3727-3745 (2010).

114. Lande R. Genetic variation and phenotypic evolution during allopatric speciation. The American Naturalist 116, 463-479 (1980).

115. Valentine JW. Biotic diversity and clade diversity. In: Phanerozoic Diversity Patterns (ed^(eds Valentine JW). Princeton University Press (1985).

116. Lamb HH. Climate: Present, Past and Future. Fundamentals and Climate Now (1972).

117. Prentice KC. Bioclimatic distribution of vegetation for general circulation model studies. JGR 95, 11811-11830 (1990).

118. Jenkins CN, Pimm SL, Joppa LN. Global patterns of terrestrial vertebrate diversity and conservation. Proceedings of the National Academy of Sciences 110, E2602E2610 (2013). 
119. Pimm SL, et al. The biodiversity of species and their rates of extinction, distribution, and protection. Science 344, 1246752 (2014).

120. Hijmans RJ. raster: Geographic Data Analysis and Modeling. R package version 2.58. https://CRANR-projectorg/package=raster, (2016).

121. Alt H, Godau M. Computing the Frechet distance between two polygonal curves. International Journal of Computational Geometry \& Applications 5, 75-91 (1995).

1204

122. Toohey K. SimilarityMeasures: Trajectory Similarity Measures. R package version 1.4. (2015). 